\title{
Assistive Technology for Fall Detection Development of Integrated Wearable Sensor to Smart Home System
}

\author{
Guilherme Gerzson Torres; Yuri das Neves Valadão; Tiago Rodrigo Cruz; Ivan Müller \\ Electrical Engineering Post Graduation Program \\ Universidade Federal do Rio Grande do Sul \\ Porto Alegre, Brazil \\ Email: guistacruz@gmail.com; yuri.nv@gmail.com; tiago.cruz@ufrgs.br; ivan.muller@ufrgs.br
}

\begin{abstract}
Fall detection is an assistive technology for elderly people that helps in emergency situations. This work presents the development of a wearable device to detect falls connected to a ultra low power wireless network. The device is connected to a smart home system to trigger alarms when events are detected. The fall detection is done by a threshold algorithm based on data fusion from inertial sensors. The wearable sensor is based on EnOcean protocol, which includes a wireless connection with a smart home system, according to the KNX standard, through the Home Assistant platform. The tests were performed in a prototype and the results include the evaluation of fall and nonfall movements in two different body characteristics. The results revealed sensitivity and specificity of up to $96 \%$ and $100 \%$, respectively.
\end{abstract}

Index Terms-EnOcean, KNX, Wearable sensor, Sensor Fusion, Fall Detection

\section{INTRODUCTION}

Recently, several socioeconomic factors promoted researches on assistive technologies, including the ones related to elderly people. Currently, $40 \%$ of the elderly live independently worldwide [1]. Therefore, the use of assistive technologies has been treated as an important topic in the area of assistance for this large part of the population. It is possible to imagine that in the future, because of the aging of the world population, the problems faced today in the areas of health and medical care will increase, demanding new solutions.

Moreover, the increasing technological advance makes it possible to create smart home environments increasing energy monitoring, comfort and, consequently, improving people's quality of life [2]. The relationship of these smart homes with the growth of the elderly population can be interconnected, comfortably and smoothly.

The earlier detection of an emergency increases the chances that the elderly will have appropriate and agile treatment, cooperating for various factors such as reduction of sequelae, hospital expenses and medical hospitalizations. Therefore, the importance of identifying related emergencies should be considered. When incorporated to a smart house network, these technologies can provide a continuous assistance, since they can provide services such as remote medical care and monitoring, as well as providing greater independence and better quality of life, given that the elderly prefer to be in the comfort of their homes instead of treatment centers and hospitals. The identification of falls by means of noninvasive as much as possible methods has been done through the development of wearable devices equipped with inertial sensors, using fall detection algorithms.

Thereby, concerning studies on assistive technologies for fall detection, the following important gaps were found. These are related to the development of algorithms for effective detection of falls based on inertial sensors. The algorithm must be computationally efficient, and low power consumption in order to be incorporated in wireless sensors. Such algorithms must present efficient results, with the minimum of false detection to increase reliability. It is also important to integrate such fall sensors into smart house networks. Therefore, the objective of this work is the development of a wearable prototype device, using ultra low power wireless technology where alarm messages serves as an active sensor in the ecosystem of a home automation system [3].

\section{RELATED WORKS}

Modern medicine allows people to live healthier for longer when compared to previous generations. In fact, it is estimated that $20 \%$ of the world population will be over 65 in 2050 . The aging of the population poses challenges both for society, such as inclusion and accessibility, and for health systems, such as availability of doctors and medicines. As people age, they become more susceptible to disease and disability and consequently they have to face difficulties in their daily life. These difficulties are often due to chronic diseases, problems with vision and deafness, physical and cognitive limitations. Considering the fact that most elderly people choose to live in their own homes, the development of Ambient Assisted Living (AAL) technologies is being considered of great importance, since it promotes greater independence and improves the quality of life of the elderly [4].

According to [5], there are three generations of AAL technologies. The first generation consists of personal alarm devices, such as alarm buttons where the elderly, when necessary, press it to make contact with someone to act 
according to the type of emergency. The second generation consists of more elaborated devices which detect potential emergency cases automatically through sensors strategically placed in the environment, such as cameras, allowing a more efficient monitoring of the elderly. An advantage of this type of approach is the detection of emergency cases without requiring the elderly to act by pressing a button, for example, but it raises doubts related to users' privacy. The third generation is characterized by developing an intelligent environment to detect and report problems or emergencies in a less invasive manner. These systems are characterized by integrating computers and devices into routines in order to monitor both the environment and the elderly. Wearable devices and other sensors installed in the environment serve to monitor vital signs and user routine patterns. Actuators, arranged in the environment, assist the elderly by facilitating the use of devices such as example, air conditioning and appliances. Therefore, it can be considered that the innovations in AAL technologies are directly linked to the constant evolution of technologies in the areas of smart homes, robotics and sensors.

In the literature, many works use wearable devices to detect falls, and they are characterized by using sensors to detect posture and body movements. The main approaches to fall detection using wearable devices can be classified as thresholdbased systems and machine learning-based systems [6]. Classification forms are used to distinguish various movements from falling situations, including K-Nearest Neighbors (KNN), Support Vector Machine (SVM), Hidden Markov Method (HMM) machine learning techniques, or by observing movements with the purpose to generate algorithms that consider boundaries [7], [8].

The method of limiting algorithms is based on determining limits based on motion data to differentiate between different movements, in other words, these limits serve to differentiate between a falling and a non-falling movement, as each type of movement generates a type of data related to it. Therefore,it is possible to differentiate movements based on some data aspect, thus generating a proper limit threshold. From testing, these limits can be determined using machine learning observation or automatic classification techniques such as K-NN, SVM and HMM. Generally, inertial sensors are employed due to low cost and convenience, as falls are usually related to variations in body acceleration, making the use of accelerometers pertinent. In turn, gyros can be used to improve systems accuracy by analyzing angular velocity in falling movements.

[9], using an algorithm based on data from two sensors equipped with 3-axis accelerometer and gyroscope, arranged in different positions in the body, identifying postures and movements. [10] and [11], presented monitoring systems that detect falls through boundary analysis algorithms, using accelerometers to determine the acceleration vector sum and body angle for the $\mathrm{XY}$ plane, determining the acceleration phases during fall, impact and the horizontal position of the body after impact. [12], developed an algorithm whose data from 3-axis gyro and accelerometer are used to the vector sum of angular velocity acceleration signals as limits for analysis.
[13], through acceleration analysis, uses SVM to determine the boundaries of the algorithm, separating falling movements from normal activity movements. [14] and [15], use 3-axis accelerometers to collect data related to falls to use HMM and to assess the risks of falling. [7] and [8], use 3-axis accelerometer and/or gyro data and a K-NN algorithm to differentiate normal activities from falls.

It is virtually impossible to compare the performance of different fall detection methods as there are no established evaluation criteria or test methods. To circumvent this fact, a quality assessment system is based on two concepts: (i) sensitivity, which is the ability of the sensor to detect falls, in others words, evaluates performance against false negatives, and (ii) specificity, which is the ability to detect only fall, that is, evaluates the performance regarding false positives (proposed by [16]). They are determined according to the following equations, where (1) is related with sensitivity, and (2) is related with specificity.

$$
\begin{aligned}
& \text { Sensitivity }=\frac{\text { true positive }}{\text { true positive }+ \text { false negative }} \\
& \text { Specificity }=\frac{\text { true negative }}{\text { true negative }+ \text { false positive }}
\end{aligned}
$$

The present work uses a complementary filter to detect falls. This filter is suitable for applications where the noise is not easily determined, it uses two measurements from different sources, where one of the sources can be more suitable for low frequency region and the other one for high frequency region. Besides that, the work proposes a connection with AAL through KNX technology, which can send alert messages to registered people, notifying the user's fall. This work is also characterized by the low power consumption and energy harvesting provided by the EnOcean technology.

\section{Methods AND MATERIALS}

For the development of the proposal, the following methods and materials were employed. The wearable device uses EnOcean technology, which is a well established technology for ultra low power wireless devices, being adequate for the wearable ones [17]. In its firmware, there is a fall detector algorithm using the limits analysis, based on data from inertial sensors (accelerometers and gyroscope). The wearable device is integrated with the KNX home automation network, through the Home Assistant platform. Once connected to a KNX network, the developed device acts as a fall detector, enabling the emergency alarm.

EnOcean technology, promoted by the EnOcean Alliance, is an emerging technology that allows the use of radio transceivers without the use of batteries. This is an optimization solution based on ultra low energy consumption and energy harvesting. Energy harvesting results from wireless sensors that acquire energy from movement, pressure, light or temperature variation, converting them for using and storing it 
in sets of small batteries or supercacitors. EnOcean operates in the $868 \mathrm{MHz}$ and $315 \mathrm{MHz}$ frequencies and supports a transmission range up to $30 \mathrm{~m}$ indoors and $300 \mathrm{~m}$ outdoors [18], [19].

A KNX network can be used in intelligent house systems [20]. KNX is also a well established technology for home automation systems, consequently, considered a good choice for this proposal. The KNX standard features a decentralized control system, allowing all of its components, sensors or actuators, to have their own controller, which permits them to be programmed according to the application. The KNX communication system presents a low-speed transfer system, a feature that allows the system to have a high tolerance to noise and the ability to respond to physical changes in the environment. Its low speed limits the ability to implement the standard for industrial level machine control that is why their approach is usually housing [21]. The EnOcean system is integrated with the KNX system through the Home Assistant platform.

The connection of the KNX system with the ETS software was established by the KNX IP Router device, allowing connection to the system via wired or wireless technology. The rest of the KNX devices are connected to the KNX IP router through twisted pair cables forming a drop-in network topology for the multiple sensors. The system is programmed using the ETS software S-mode, which is connected to the router via Ethernet cable. Fig. 1 provides an overview of system integration. The wearable device sends alarm messages to an EnOcean data collector that is connected to a Raspberry PI, which is a Single Board Computer (SBC). The Home Assistant application runs on SBC and data is sent to KNX system by Ethernet cable.

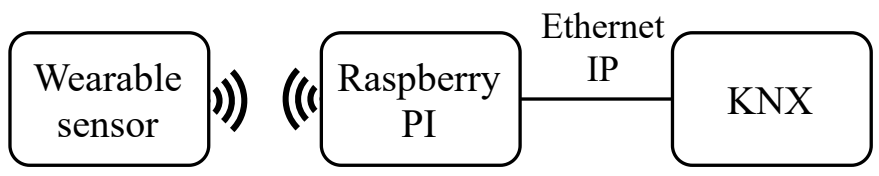

Figure 1. Overview of the system.

\section{A. Wearable Sensor Development}

The EOP350 development system is used to program an EnOcean STM300 device. The STM300 is an EnOcean RF transceiver module that enables the development of sensors and wireless actuators, presenting ultra low power consumption. It has an integrated MCU with $2 \mathrm{kB}$ SRAM and up to 16 configurable I/O ports, with 10-bit ADC and 8-bit DAC. The RF transceiver operates at $868.300 \mathrm{MHz}$ frequency with ASK modulation.Firmware programming is done through the use of the Dolphin API library, using C language through Keil's $\mu$ Vision software. The inertial sensor used is MPU-9250 from InvenSense, a widely used sensor in industry. It presents suitable sensing for wearable devices, witch is composed by 3 axis accelerometer, 3-axis gyroscope and 3-axis magnetometer. The full scale ranges can be chosen from $\pm 2 \mathrm{~g}$ to $\pm 16 \mathrm{~g}$ in the accelerometer, and between $\pm 250^{\circ} / \mathrm{s}$ up to $\pm 2000^{\circ} / \mathrm{s}$ in the gyroscope. Both sensors have 16-bit ADC and communicate with the STM300 module is via $\mathrm{I}^{2} \mathrm{C}$.

To function as a wearable fall detector device, the device is coupled to user's chest so that the horizontal plane is the $\mathrm{XZ}$ plane. Fig. 2 presents the prototype under test.

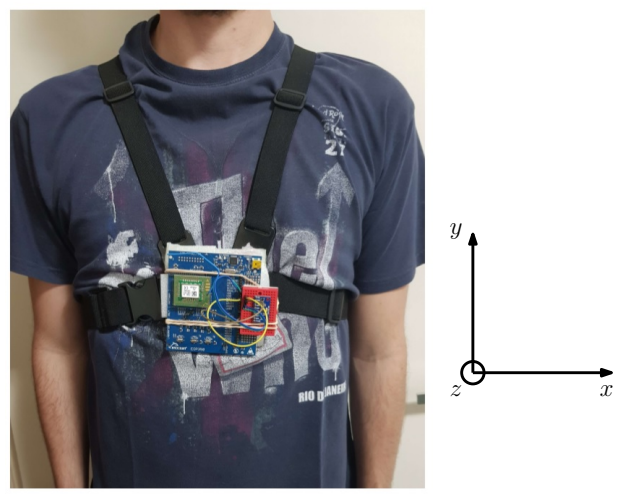

Figure 2. Prototype attached to user's chest

\section{B. KNX Network Integration}

The integration between the wearable device and the KNX system is done through home assistant which is an open home automation platform source code developed in Python, supporting various technologies, including KNX and EnOcean. Home automation occurs through the interpretation of devices, where the user can configure commands for home control. The Home Assistant platform is installed on a Raspberry PI 3 SBC. Linux based operating systems runs on Cortex-A53, 64-bit quad-core 1.2GHz processor, with 1GB RAM, 802.11n and Bluetooth wireless connections [22].

For the Home Assistant configuration, it is necessary to establish a connection to KNX system and EnOcean sensor. The $\mathrm{KNX}$ connection is via IP port and EnOcean sensor connection is via the USB300 EnOcean device, which connects to a USB port for the purpose of interpreting EnOcean telegrams. In this work, it is defined a specific ID for the EnOcean sensor, when sending the fall alert telegram, which interacts with a KNX group address. This address refers to one of the actuator relays of the KNX system.

An Android smartphone is used as a centralized control unit and works connecting to the KNX system wirelessly. Application setup is performed through the AyControl Editor software. This software is widely used in home automation systems, employed to provide remote control.

\section{Fall Detection Algorithm}

In order to provide an effective fall detection, four fall and four non-fall movements are suggested and analyzed: standing, walking, sitting on a chair, running, back/forward falls and left/right falls. Fall movements are shown in Fig. 3.

Each movement is recorded based on four types of signals: acceleration, angular velocity, and inclination on the $X$ and $\mathrm{Z}$ axes. The inclination in the $\mathrm{X}$ and $\mathrm{Z}$ axes are considered 


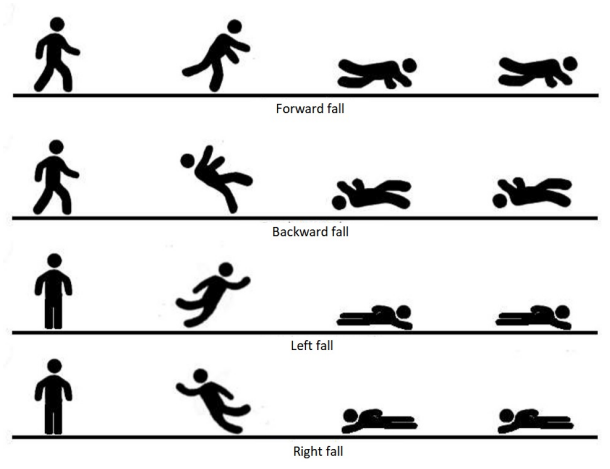

Figure 3. Considered fall movements

from $-90^{\circ}$ to $90^{\circ}$, being $0^{\circ}$ position when the body is vertical, being able to tilt from $-90^{\circ}$ to $90^{\circ}$ on both $\mathrm{X}$ and $\mathrm{Z}$ axes. Accelerometer and gyroscope produce values of acceleration (in g) and velocity angles (in $\%$ s), respectively, in each axis, as well as the RMS (Root Mean Square) values.

Three methods of obtaining the angular values of the inclinations in the $\mathrm{XZ}$ plane are used. The first method is a simplified estimation that considers the raw data from the sensor divided by a constant, determined by the configured resolution. The second method is the estimation that uses the arc tangent, implemented by using atan 2 function, to avoid indeterminations, considering the four quadrants. The third method deals with obtaining the angle derived from merging accelerometer and gyroscope sensors data through the complementary filter, as shown in Fig. 4.

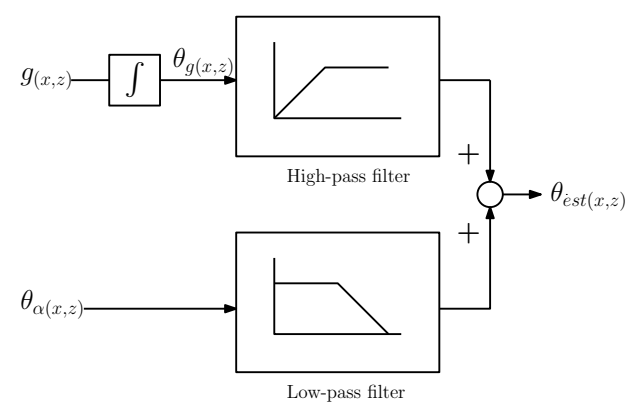

Figure 4. Block diagram of a complementary filter

The second method of angle determination is used by reading accelerometer data as the input $\theta \alpha_{x z}$. To determine $\theta g_{x z}$ it is necessary to carry out the integral of the gyroscope on the $\mathrm{X}$ and $\mathrm{Z}$ axis of the sensor. The integration of gyroscope measurements leads to drift, so it is necessary to configure the filter's high-frequency cutoff in order to eliminate any drifting. The equation (3) is used to obtain filter's parameters. A value $\Delta \mathrm{t}=0.035 \mathrm{~s}$ is established considering the sampling time of the sensor signals. To determine the $\gamma$ value, tests are performed in relation to filter performance. The value that presented better performance was $\gamma=0.8$, where the influence of the drift becomes negligible in the signal. With this value, it is possible to determine the $\alpha$ parameter of the filters using the following equation:

$$
\gamma=\frac{\alpha}{\Delta t-\alpha}
$$

The estimated angle of inclination of the $\mathrm{XZ}$ plane $\left(\hat{\theta}_{k}\right)$ is defined by the recursive equation given by (4), where $w_{k}$ is the data acquired by the gyroscope.

$$
\hat{\theta}_{k}=(1-\gamma) \theta_{a, k}+\gamma\left(\hat{\theta}_{k-1}+\Delta t w_{k}\right)
$$

Therefore, (5) is used recursively by the system, based in (4), to acquire the inclination value of the angle in the $X$ axes and in the $\mathrm{Z}$ axes.

$$
\hat{\theta}_{k}=0.2 \theta_{a, k}+0.8\left(\hat{\theta}_{k-1}+0.035 w_{k}\right)
$$

The use of the supplementary filter was considered since it is a suitable to be used in devices with limited computational capacity, as is the case of 8051 microcontroller embedded in the STM300 EnOcean module.

\section{CAse Study}

In order to evaluate the proposed system, falls and nonfall movements were performed by a male individual, $1.75 \mathrm{~m}$ height and $70 \mathrm{~kg}$ and a female, $1.60 \mathrm{~m}$ height and $66 \mathrm{~kg}$.

From data produced by the tests, it is possible to perform the graphical analysis of the movements, to determine the limits to be considered in the algorithm.

Fig. 5 shows the graphs obtained from movements standing, walking, sitting on a chair and running. Fig. 6 shows data obtained from a forward fall. The graphs representing the inclinations in the XZ plane are formed by 3 signals, where each line represents different methods of obtaining the angle of inclination. The blue line represents the most simplified form of estimation, considering raw data divided by the set resolution. The yellow line represents the method using arc tangent. Both methods present noise, because data are due to accelerometer signals only. The green line represents the angle derived from the data fusion of accelerometer and gyro sensors through the complementary filter.

From the obtained data, the limits to be analyzed by the fall detection algorithm are established. To identify a fall, the RMS acceleration values, linear and angular velocity, and inclination in degrees in the $\mathrm{X}$ and $\mathrm{Z}$ axes are considered. As shown in Fig. 5, the linear acceleration only exceeds $2 \mathrm{~g}$ in the impact, but the RMS value of the angular velocity never exceeds the value of $200 \%$, and the angular inclination in both axes always remained at levels that exceed $40^{\circ}$. On the other hand, the analysis of the fall graphs reveals that the linear acceleration and the angular velocity are the first exceeded limits, exceeding values of $2 \mathrm{~g}$ and $200^{\circ} / \mathrm{s}$, respectively. Therefore, the algorithm considers these values as the first two limits. After reaching significant values, the algorithm analysis the inclination during 5 seconds. For both forward/backward, plane YZ, and right/left falls, plane XY, the stipulated limits are $-70^{\circ}$ and $70^{\circ}$. In this way, it is possible to use these threshold values to determine when the elderly 


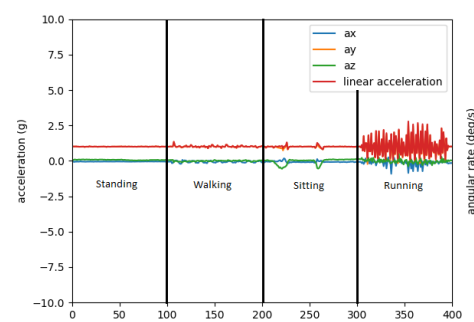

(a)

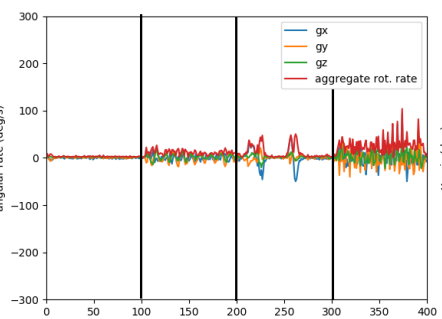

(b)

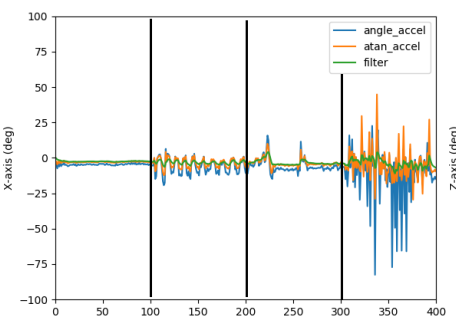

(c)

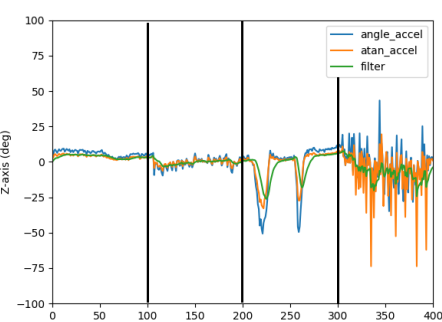

(d)

Figure 5. Non-fall movements, data relating to (a) Acceleration, (b) Angular velocity, (c) Inclination on the $\mathrm{X}$ axis and (d) Inclination on the Z axis

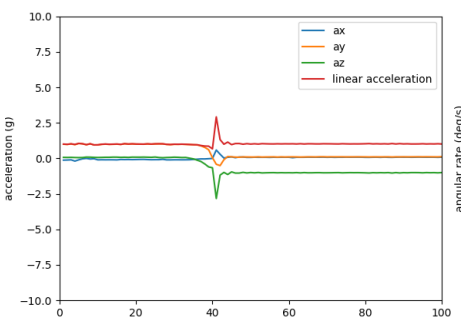

(a)

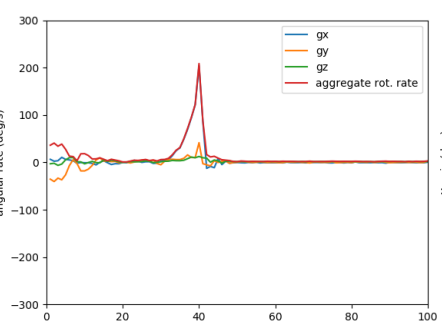

(b)

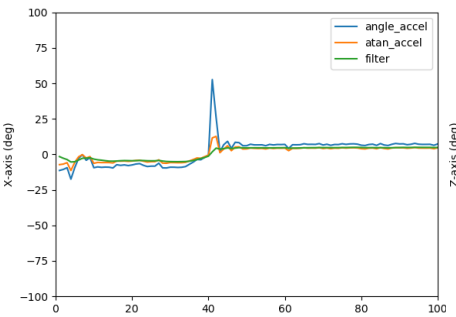

(c)

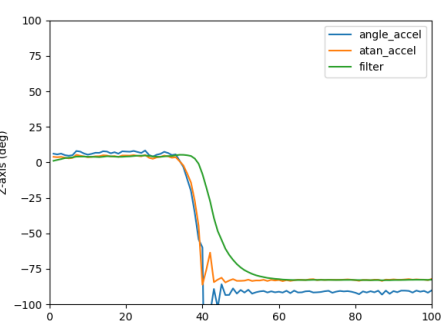

(d)

Figure 6. Movements falling forward, data concerning to (a) Acceleration, (b) Angular velocity, (c) Inclination on the $\mathrm{X}$ axis and (d) inclination on the $\mathrm{Z}$ axis

person is lying on the floor. The range of 5 seconds is enough to analyze the user's position as soon as the first limits are exceeded. Based on the values of established limits.

\section{RESULTS}

This work presented a fall detection system for monitoring the elderly, integrated to the KNX home automation protocol. As the comparison between fall detectors is difficult due to the various methods used, a quality assessment system is based on two concepts: sensitivity, which is the sensor's ability to detect falls, that is, to evaluate performance against false negatives, and specificity, which is the ability to detect only the fall, that is, it evaluates the performance in relation to false positives [16]. This idea is part of the concept of a confusion matrix with two columns and two lines informing the number of false positives and negatives, and positives and negatives, and allows the analysis of the sensitivity and specificity of the algorithm. Table I presents the references used as a comparison and Table II, the results of two sets of tests performed with the developed prototype, for each of the two individuals (case 1 and 2 ).

Table I

PREVIOUS WORKS SELECTED FOR COMPARISON.

\begin{tabular}{|c|c|c|c|}
\hline Author & Sensor & Method & Sens./Spec. \\
\hline$[10]$ & Accelerometer & Threshold algorithm & $97 \% / 98 \%$ \\
\hline$[11]$ & Accelerometer & Threshold algorithm & $96 \% / 97 \%$ \\
\hline$[9]$ & $\begin{array}{c}\text { Two accelerometers } \\
\text { and gyroscope }\end{array}$ & Threshold algorithm & $91 \% / 92 \%$ \\
\hline$[13]$ & Accelerometer & $\begin{array}{c}\text { Threshold algorithm } \\
\text { support vector machine }\end{array}$ & $100 \% /-$ \\
\hline$[8]$ & $\begin{array}{c}\text { Accelerometers } \\
\text { and gyroscope }\end{array}$ & $\begin{array}{c}\text { k-nearest neighbor } \\
\text { algorithm }\end{array}$ & $97 \% / 97,5 \%$ \\
\hline$[14]$ & Accelerometer & $\begin{array}{c}\text { Hidden Markov } \\
\text { algorithm }\end{array}$ & $100 \% / 100 \%$ \\
\hline Prop. System & $\begin{array}{c}\text { Accelerometers } \\
\text { and gyroscope }\end{array}$ & $\begin{array}{c}\text { Complementary } \\
\text { Filter }\end{array}$ & $96 \% / 100 \%$ \\
\hline
\end{tabular}

Table II

RESULTS FROM THIS PROPOSAL.

\begin{tabular}{|c|c|c|}
\hline Movement & Alarms(case 1) & Alarms(case 2) \\
\hline Walk & $0 / 100$ & $0 / 100$ \\
\hline Jump & $0 / 100$ & $0 / 100$ \\
\hline Sit & $0 / 100$ & $0 / 100$ \\
\hline Run & $0 / 100$ & $0 / 100$ \\
\hline Forward fall & $98 / 100$ & $84 / 100$ \\
\hline Backward fall & $97 / 100$ & $87 / 100$ \\
\hline Right fall & $94 / 100$ & $87 / 100$ \\
\hline Left fall & $98 / 100$ & $85 / 100$ \\
\hline
\end{tabular}

The first test was performed by a male user, 27 years old, $1.75 \mathrm{~m}$ tall and weight of $70 \mathrm{~kg}$. The second test was performed by a female user, 62 years old, $1.60 \mathrm{~m}$ tall and weight of $70 \mathrm{~kg}$. The tests were performed with two users with different body characteristics, in order to determine whether the difference in these characteristics influenced the performance of the device. The experiment considers eight types of movements: standing, walking, sitting in a chair, running, falls backward, forward, left and right. Each movement was repeated 100 times.

When movements, which are not characterized as falls, were performed by both individuals, no false positive was detected, demonstrating a superior performance when compared to the references. However, some false negatives happened. In the first test, the algorithm reached average values of sensitivity and specificity of $96 \%$ and $100 \%$, respectively. In the second test, the algorithm achieved lower performance. This is due to the fact that the calibration values for the algorithm where obtained through the case 1 tests, revealing the dependence of body characteristics in the determination limit values. 
However, it is possible to highlight that, due to the fact that using data from the inertial sensors through data fusion, the device is accurate in the detection of the body inclination and, therefore, it avoids the occurrence of false alarms because it limits the effect of noise on the signal. The detection of true positives, therefore needs to be improved through a calibration that considers the characteristics of each type of user.

\section{CONCLUSIONS}

Even with the results presented, the prototype is not perfect, generating measurement errors. These errors can be associated with different coporal characteristics, as mentioned earlier. Therefore, a possibility for future work is to add an algorithm calibration method based on different body characteristics, making it possible to improve these errors. Still, for the purpose of future work, we have the intention of miniturizing hardware, making it the least invasive as possible. This miniaturization also makes it possible to make better use of EnOcean technology, promoting greater system autonomy. Finally, even with the use of communication from the KNX protocol, it is thought that it is possible to make the system compatible with other home automation protocols.

\section{ACKNOWLEDGMENTS}

This study was financed in part by the Coordenação de Aperfeiçoamento de Pessoal de Nível Superior - Brasil (CAPES) - Finance Code 001 and by the Conselho.

\section{REFERENCES}

[1] R. Lee, J. Luo, C. H. Le, P. A. Phan, T. Le, and M. H. Le, "Assistive technologies for the older people: Physical activity monitoring and fall detection," in International Conference on the Development of Biomedical Engineering in Vietnam. Springer, 2017, pp. 661-665.

[2] F. Baig, S. Beg, M. F. Khan, and S. J. Nawaz, "A Method to Control Home Appliances Based on Writing Commands Over the Air," Journal of Control, Automation and Electrical Systems, vol. 26, no. 4, pp. 421-429, 2015. [Online]. Available: http://dx.doi.org/10.1007/s40313015-0184-4

[3] H. Wang, D. Zhang, Y. Wang, J. Ma, Y. Wang, and S. Li, "Rt-fall: A real-time and contactless fall detection system with commodity wifi devices," IEEE Transactions on Mobile Computing, vol. 16, no. 2, pp. 511-526, 2016.

[4] P. Rashidi and A. Mihailidis, "A survey on ambient-assisted living tools for older adults," IEEE journal of biomedical and health informatics, vol. 17, no. 3, pp. 579-590, 2012.

[5] S. Blackman, C. Matlo, C. Bobrovitskiy, A. Waldoch, M. L. Fang, P. Jackson, A. Mihailidis, L. Nygård, A. Astell, and A. Sixsmith, "Ambient assisted living technologies for aging well: a scoping review," Journal of Intelligent Systems, vol. 25, no. 1, pp. 55-69, 2016.

[6] A. Ramachandran and A. Karuppiah, "A survey on recent advances in wearable fall detection systems," BioMed Research International, vol. 2020, 2020.

[7] F. Darko, S. Denis, and Ž. Mario, "Human movement detection based on acceleration measurements and k-NN classification," EUROCON 2007 - The International Conference on Computer as a Tool, pp. 589-594, 2007.

[8] J. He, M. Zhou, X. Wang, and Y. Han, "A wearable method for autonomous fall detection based on Kalman filter and k-NN algorithm," Proceedings - 2016 IEEE Biomedical Circuits and Systems Conference, BioCAS 2016, pp. 420-423, 2016.

[9] Q. Li, J. A. Stankovic, M. A. Hanson, A. T. Barth, J. Lach, G. Zhou et al., "Accurate, fast fall detection using gyroscopes and accelerometerderived posture information." in $B S N$, vol. 9, 2009, pp. 138-143.
[10] F. Wu, H. Zhao, Y. Zhao, and H. Zhong, "Development of a wearablesensor-based fall detection system," International journal of telemedicine and applications, vol. 2015, p. 2, 2015.

[11] S. Abdelhedi, R. Bourguiba, J. Mouine, and M. Baklouti, "Development of a two-threshold-based fall detection algorithm for elderly health monitoring," in 2016 IEEE Tenth International Conference on Research Challenges in Information Science (RCIS). IEEE, 2016, pp. 1-5.

[12] H. W. Guo, Y. T. Hsieh, Y. S. Huang, J. C. Chien, K. Haraikawa, and J. S. Shieh, "A threshold-based algorithm of fall detection using a wearable device with tri-axial accelerometer and gyroscope," in 2015 International Conference on Intelligent Informatics and Biomedical Sciences (ICIIBMS). IEEE, 2015, pp. 54-57.

[13] G. N. Swathi and M. Amarnadh, "Threshold Based Fall Detection and PredictionMethod Using Tri-Axial Accelerometer," International Journal of Innovative Research in Computer and Communication Engineering, vol. 2, no. 4, pp. 29-33, 2014.

[14] L. Tong, Q. Song, Y. Ge, and M. Liu, "Hmm-based human fall detection and prediction method using tri-axial accelerometer," IEEE Sensors Journal, vol. 13, no. 5, pp. 1849-1856, 2013.

[15] D. Lim, C. Park, N. H. Kim, S.-H. Kim, and Y. S. Yu, "Fall-detection algorithm using 3-axis acceleration: combination with simple threshold and hidden markov model," Journal of Applied Mathematics, vol. 2014, 2014.

[16] N. Noury, A. Fleury, P. Rumeau, A. Bourke, G. Ó. Laighin, V. Rialli, and J. Lundy, "Fall detection - Principles and Methods," International Conference of the Engineering in Medicine and Biology Society (EMBS), vol. $29,2007$.

[17] EnOcean. (2020) Enocean: Ultra-low power management. [Online]. Available: https://www.enocean.com/en/technology/energy-harvestingwireless/

[18] P. Rawat, K. D. Singh, H. Chaouchi, and J. M. Bonnin, "Wireless sensor networks: a survey on recent developments and potential synergies," The Journal of supercomputing, vol. 68, no. 1, pp. 1-48, 2014.

[19] L. Parsons, R. Ross, and K. Robert, "A survey on wireless sensor network technologies in pest management applications," SN Applied Sciences, vol. 2, no. 1, p. 28, 2020.

[20] KNX-Association. (2020) Serial data transmission and knx protocol documentation. [Online]. Available: https://www.knx.org/knx-en/forprofessionals/training/books-documentation/index.php

[21] S. A. Navarro-Tuch, M. R. Bustamante-Bello, A. Molina, J. IzquierdoReyes, R. Avila-Vazquez, J. L. Pablos-Hach, and Y. Gutiérrez-Martínez, "Inhabitable space control for the creation of healthy interactive spaces through emotional domotics," International Journal on Interactive Design and Manufacturing (IJIDeM), vol. 12, no. 4, pp. 1337-1347, 2018.

[22] Raspberry Pi Foundation, "Raspberry Pi 3 Model B+ 1Gb," 2015, Accessed Oct 10 2019. [Online]. Available: https://static.raspberrypi.org/files/product-briefs/RaspberryPi-Model-Bplus-Product-Brief.pdf 